\title{
Large-scale structure studies with AGN in the eROSITA/SRG All-Sky Survey
}

\author{
Alexander Kolodzig ${ }^{1}$, Marat Gilfanov ${ }^{1,2}$, Gert Hütsi ${ }^{1,3}$ \\ and Rashid Sunyaev ${ }^{1,2}$
}

\author{
${ }^{1}$ Max-Planck-Institut für Astrophysik (MPA), \\ Karl-Schwarzschild-Str. 1, D-85741 Garching, Germany, alex@mpa-garching.mpg.de \\ ${ }^{2}$ Space Research Institute (IKI), Russian Academy of Sciences, \\ Profsoyuznaya ul. 84/32, Moscow, 117997 Russia \\ ${ }^{3}$ Tartu Observatory, Tõravere 61602, Estonia
}

\begin{abstract}
The four-year X-ray all-sky survey (eRASS) of the eROSITA telescope aboard the Spektrum-Roentgen-Gamma satellite will detect $\sim 3$ million active galactic nuclei (AGN) with a median redshift of $z \approx 1$. We show that this unprecedented AGN sample, complemented with redshift information, will supply us with outstanding opportunities for large-scale structure research. For the first time with a sample of X-ray selected AGN, it will become possible to perform detailed redshift- and luminosity-resolved studies of the linear bias factor, and to convincingly detected baryonic acoustic oscillations (BAOs). To exploit the full potential of the eRASS AGN sample, photometric and spectroscopic surveys of large areas and a sufficient depth will be needed.
\end{abstract}

\section{Introduction}

Large-scale structure (LSS) studies are an important tool for studies in two major areas of astrophysics: cosmology, and galaxy evolution. A key of their success is the increasing number of surveys at different wavelengths with increasing depths and sky coverages. In X-rays, many deep, extragalactic surveys have been performed in the past decade (Brandt \& Hasinger 2005; Cappelluti et al. 2012; Krumpe et al. 2013). However, in comparison with other wavelengths, X-ray surveys with a large sky coverage and sufficient depth are still rare. The four-year X-ray all-sky survey (eRASS) of the eROSITA telescope (Predehl et al. 2010) aboard the Spektrum-Roentgen-Gamma (SRG) satellite will improve this situation dramatically. The survey will detect $\sim 3$ million active galactic nuclei (AGN) with a median redshift of $z \approx 1$ and a typical luminosity of $L_{0.5-2.0 \mathrm{keV}} \sim 10^{44} \mathrm{erg} \mathrm{s}^{-1}$. In our previous work (Kolodzig et al. 2013b), we have studied the statistical properties of this AGN sample and will adopt these results here. In this work we explore the potential of studying LSS with the AGN sample to be detected in soft-energy band $(0.5-2.0 \mathrm{keV})$ on the extragalactic sky $\left(|b|>10^{\circ}, \sim 34100 \mathrm{deg}^{2}\right)$ of eRASS, which is explained in full detail in Kolodzig et al. (2013a). We focus on two important aspects of LSS studies: the clustering strength (represented by the linear bias factor, Sect. 2) and the baryonic acoustic oscillations (BAOs, Sect. 3).

In the following calculations we assumed the four-year average sensitivity of $1.1 \times$ $10^{-14} \mathrm{erg} \mathrm{s}^{-1} \mathrm{~cm}^{-2}$ computed in Kolodzig et al. (2013b, Table 1) and that redshifts are available for all eRASS AGN. We will explore the effects of redshift-errors in a forthcoming paper (Hütsi et al. 2014). 

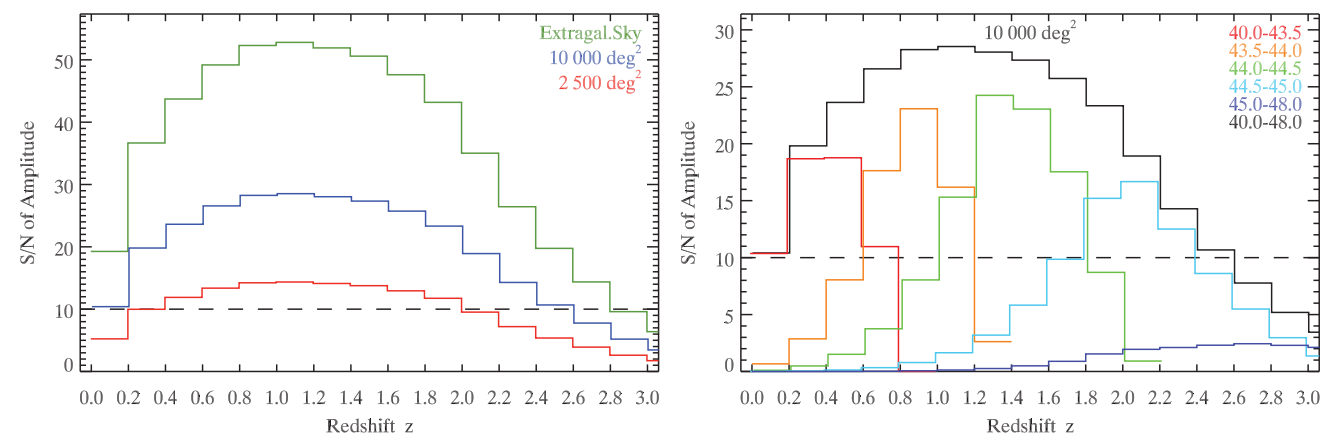

Figure 1. Signal-to-noise ratio of the amplitude of the angular power spectrum as a function of redshift (binning $\Delta z=0.2$ ). Left: For different sky fractions. Right: For different luminosity intervals (in units of $\left.\log _{10}\left(L\left[\mathrm{erg} \mathrm{s}^{-1}\right]\right)\right)$ and a sky coverage of $10000 \mathrm{deg}^{2}$.

\section{Linear bias factor}

The linear bias factor $b$ provides a simple and direct estimate of the average mass of dark matter halos (DMHs) that host a given subpopulation of AGN. Measurements of this quantity suggest a higher DMH mass for AGN than for quasars for $z \geqslant \sim 1$ and a weak correlation between DMH mass and AGN luminosity for $z \sim 0.5$ (e.g. Krumpe et al. 2010, 2012; Miyaji et al. 2011; Allevato et al. 2011, 2012; Cappelluti et al. 2012; Mountrichas \& Georgakakis 2012; Mountrichas et al. 2013; Koutoulidis et al. 2013; Fanidakis et al. 2013). However, uncertainties are still large and AGN luminosities available for these studies are typically limited by $L \sim 10^{44} \mathrm{erg} \mathrm{s}^{-1}$. With more accurate measurement of the behavior of the linear bias factor with redshift and luminosity and a comparison with simulations, we will be able to improve our understanding of major questions, such as the nature of the AGN environment, the main triggering mechanisms of AGN activity (e.g. Koutoulidis et al. 2013; Fanidakis et al. 2013) and how supermassive black holes co-evolve with the DMH over cosmic time (e.g. Alexander \& Hickox 2012).

The linear bias factor is measured by comparing the observed clustering strength of AGN with the theoretical clustering strength of the DMHs, under the assumptions of a certain cosmology. We predict the clustering strength of the AGN detected in eRASS using the AGN clustering model of Hütsi et al. (2012), which enables us to compute the angular power spectrum (PS) of eRASS AGN for different redshift and luminosity intervals. The PS amplitude represents the clustering strength of AGN and since it is proportional to the square of the linear bias factor of AGN $\left(\propto b^{2}\right)$, its uncertainty (e.g. Kolodzig et al. 2013a, Eq. 5) directly reflects the uncertainty of measuring the latter.

In Fig. 1 the achievable signal-to-noise ratio $(\mathrm{S} / \mathrm{N})$ of the PS amplitude is shown as a function of redshift. We can see on the red curve for $\sim 2500 \mathrm{deg}^{2}$ in the left panel that we will be able to measure the amplitude to a high accuracy $(<10 \%)$ for a wide redshift range between $\sim 0.2$ and $\sim 2.2$ even with a fairly small fraction of the sky. Owing to the high $\mathrm{S} / \mathrm{N}$ of the PS amplitude, we will be able to separate the AGN into different luminosity groups. The right panel shows that we will be able to achieve an accuracy of $<10 \%$ for most of these groups. Therefore, it will be possible to perform a redshiftand luminosity-resolved analysis of the linear bias factor of AGN with eRASS with high statistical accuracy. Further, we will be able to expand our analysis to the luminosity range beyond $L \sim 10^{44} \mathrm{erg} \mathrm{s}^{-1}$, the luminosity domain characteristic of quasars. Thus, the eRASS data will not only increase the redshift- and luminosity resolution of DMH mass estimate of AGN, but will open possibilities for a detailed comparison of the clustering properties of luminous AGN and optical quasars. 

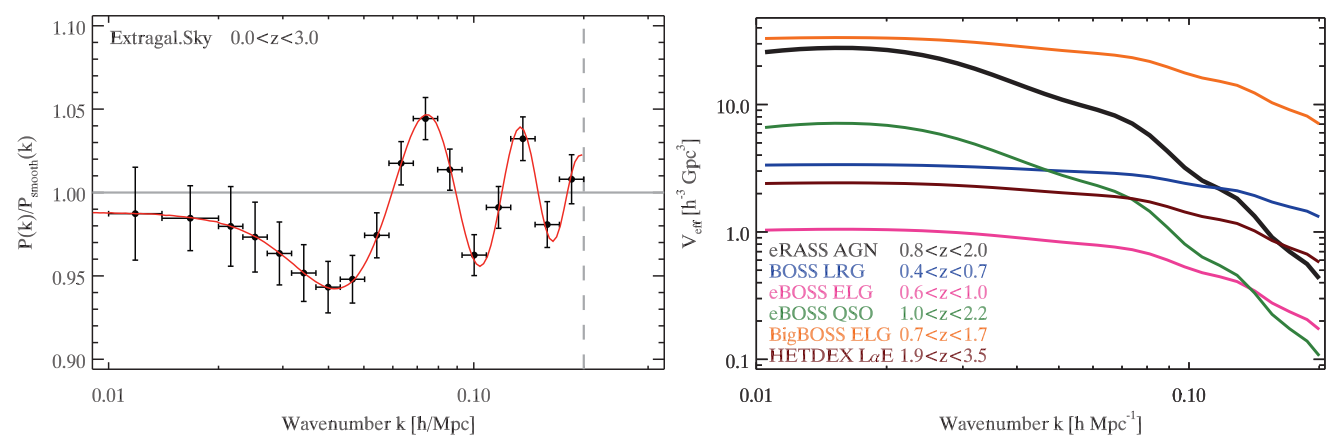

Figure 2. Left: BAOs in the spatial power spectrum for the extragalactic sky in the redshift range $0.0<z<3.0$. At wavenumbers above the vertical dashed line (corresponding to $0.2 \mathrm{~h} \mathrm{Mpc}^{-1}$ ) our assumption of a linear clustering starts to break down. The red curve shows the original input model for the BAOs. Right: Effective volumes of BAO surveys as a function of wavenumber. Effective volumes are computed for redshift ranges indicated in the plot.

\section{Baryonic acoustic oscillations}

Acoustic peaks in the power spectra of matter and CMB radiation, theoretically predicted by Sunyaev \& Zeldovich (1970); Peebles \& Yu (1970), are among the main probes for measuring the kinematics of the Universe (e.g. Weinberg et al. 2012). Galaxy surveys have recently reached sufficient breadth and depth for the first convincing detection of these acoustic peaks in the PS of matter, achieved with the SDSS data (Cole et al. 2005; Eisenstein et al. 2005; Hütsi 2006; Tegmark et al. 2006). Since then, BAOs have been measured extensively up to $z \sim 0.8$, in particular with luminous red giant galaxies (LRGs) (e.g. Anderson et al. 2012). A detection beyond $z \sim 0.8$ will be another significant milestone but has yet not been accomplished by using galaxy distributions.

For the uncharted range from $z \sim 0.8$ up to $\sim 2.0$, AGN, quasars and emission-line galaxies (ELGs) are proposed to be the best tracers for measuring BAOs. However, currently existing surveys do not achieve the required statistics for a proper detection (Sawangwit et al. 2012; Comparat et al. 2013). eRASS and eBOSS will be the first surveys to change this situation but eRASS will have by far the largest sky coverage compared to eBOSS and all other dedicated optical BAO surveys.

In the left panel of Fig. 2 we illustrate the BAO signal from the eRASS AGN sample by showing the ratio of the 3D linear PS to a smoothed PS. The PS of the AGN contains by default BAOs but for the smoothed PS the BAOs are removed (for details see e.g. Eisenstein \& $\mathrm{Hu}$ 1998). Both power spectra were computed with the AGN clustering model of Hütsi et al. (2012). The plot shows that with the whole eRASS AGN sample for the extragalactic sky we expect to be able to detect the BAOs with a confidence level of $\sim 14 \sigma$. For the currently unexplored redshift range of $0.8-2.0$ a confidence level of $\sim 11 \sigma$ will be achieved. Decreasing the sky area to $20000 \mathrm{deg}^{2}$ and $10000 \mathrm{deg}^{2}$, we obtain $\sim 8 \sigma$ and $\sim 6 \sigma$, respectively. The values quoted in Kolodzig et al. (2013a) are smaller, because the cross-spectra of the angular PS between different redshift bins were not considered in these calculations (See also Hütsi et al. 2014).

In right panel of Fig. 2 we compare the potential of measuring BAOs in eRASS and dedicated optical BAO surveys (For a complete list see Table 1 in Kolodzig et al. 2013a) by showing their effective volumes $V_{\text {eff }}$ as a function of wavenumber. The effective volume is an estimate for the statistical performance of a galaxy clustering survey (e.g. Eisenstein et al. 2005) and the uncertainty of the PS depends on it with $V_{\text {eff }}^{-0.5}$. It is remarkable to see from the plot that the statistical strength of eRASS for BAO studies is comparable 
with that of dedicated optical BAO surveys, even though eRASS was never designed for this purpose. Potentially, the eRASS AGN sample will become the best sample for BAO studies beyond redshift $z>0.8$ until the arrival of BigBOSS at the end of this decade. However, to realize this potential comprehensive redshift measurements will be required.

\section{Summary}

We have shown that eRASS with its $\sim 3$ million AGN sample will supply us with outstanding opportunities for detailed LSS research. Statistically accurate redshift- and luminosity-resolved studies of the linear bias factor will become possible for the first time, using the data from a sky area of the order of $10^{4} \mathrm{deg}^{2}$. The eRASS AGN sample will expand their luminosity range beyond $L_{0.5-2.0 \mathrm{keV}} \sim 10^{44} \mathrm{erg} \mathrm{s}^{-1}$, thus enabling a direct comparison of the clustering properties of luminous X-ray AGN and optical quasars. For the first time for X-ray selected AGN, eRASS will be able to detect BAO with high-statistical significance of $\sim 14 \sigma$. Moreover, it will push the redshift limit of BAO detections beyond the current limit of $z \sim 0.8$. However, for this potential to be realized and exploited, spectroscopic quality redshifts for large areas of the sky are required.

\section{References}

Alexander, D. M. \& Hickox, R. C. 2012, New A Rev., 56, 93

Allevato, V., Finoguenov, A., Cappelluti, N., et al. 2011, ApJ, 736, 99

Allevato, V., Finoguenov, A., Hasinger, G., et al. 2012, ApJ, 758, 47

Anderson, L., Aubourg, E., Bailey, S., et al. 2012, MNRAS, 427, 3435

Brandt, W. N. \& Hasinger, G. 2005, ARA\& $A, 43,827$

Cappelluti, N., Allevato, V., \& Finoguenov, A. 2012, Advances in Astronomy, id. 853701

Cole, S., Percival, W. J., Peacock, J. A., et al. 2005, MNRAS, 362, 505

Comparat, J., Kneib, J.-P., Escoffier, S., et al. 2013, MNRAS, 428, 1498

Eisenstein, D. J. \& Hu, W. 1998, ApJ, 496, 605

Eisenstein, D. J., Zehavi, I., Hogg, D. W., et al. 2005, ApJ, 633, 560

Fanidakis, N., Georgakakis, A., Mountrichas, G., et al. 2013, MNRAS, 435, 679

Hütsi, G. 2006, A\&广A, 449, 891

Hütsi, G., Gilfanov, M., Kolodzig, A., \& Sunyaev, R. 2014, eprint arXiv:1403.5555

Hütsi, G., Gilfanov, M., \& Sunyaev, R. 2012, A\&A, 547, A21

Kolodzig, A., Gilfanov, M., Hütsi, G., \& Sunyaev, R. 2013a, A\&ऽA, 558, A90

Kolodzig, A., Gilfanov, M., Sunyaev, R., Sazonov, S., \& Brusa, M. 2013b, A\&A, 558, A89

Koutoulidis, L., Plionis, M., Georgantopoulos, I., \& Fanidakis, N. 2013, MNRAS, 428, 1382

Krumpe, M., Miyaji, T., \& Coil, A. L. 2010, ApJ, 713, 558

Krumpe, M., Miyaji, T., \& Coil, A. L. 2013, ArXiv e-prints, 1308.5976

Krumpe, M., Miyaji, T., Coil, A. L., \& Aceves, H. 2012, ApJ, 746, 1

Miyaji, T., Krumpe, M., Coil, A. L., \& Aceves, H. 2011, ApJ, 726, 83

Mountrichas, G. \& Georgakakis, A. 2012, MNRAS, 420, 514

Mountrichas, G., Georgakakis, A., Finoguenov, A., et al. 2013, MNRAS, 430, 661

Peebles, P. J. E. \& Yu, J. T. 1970, ApJ, 162, 815

Predehl, P., Andritschke, R., Böhringer, H., et al. 2010, in Society of Photo-Optical Instrumentation Engineers (SPIE) Conference Series, Vol. 7732, Society of Photo-Optical Instrumentation Engineers (SPIE) Conference Series

Sawangwit, U., Shanks, T., Croom, S. M., et al. 2012, MNRAS, 420, 1916

Sunyaev, R. A. \& Zeldovich, Y. B. 1970, Ap\&SS, 7, 3

Tegmark, M., Eisenstein, D. J., Strauss, M. A., et al. 2006, Phys. Rev. D, 74, 123507

Weinberg, D. H., Mortonson, M. J., Eisenstein, D. J., et al. 2012, Physics Reports, 530, 87 